Int. J. Dev. Biol. 58: 793-798 (2014)

doi: $10.1387 / \mathrm{ijdb} .140308$ ho

\title{
Intracellular localizations of the Dead End protein in Xenopus primordial germ cells
}

\author{
AYAKA TAGUCHI, KENJI WATANABE and HIDEFUMI ORII* \\ Department of Life Science, University of Hyogo, Kamigori, Akou-gun, Hyogo, Japan
}

\begin{abstract}
We investigated the intracellular localization of Xenopus Dead end protein (Dnd1) in primordial germ cells during early development by expressing the tagged protein in transgenic Xenopus embryos, with the germ plasm visualized. Dnd1 initially localized to the germ plasm in the cortex, moved to the perinuclear region together with the germ plasm after the midblastula transition, and then entered the nucleus. Using Dnd1 deletion mutants, we identified two distinct but overlapping regions of Dnd1 that were responsible for localization to either the germ plasm or nucleus. These Dnd1 regions appeared to function in primordial germ cell- and stage-specific manners.
\end{abstract}

KEY WORDS: Dnd1, germline, germ plasm, nuage, nuclear localization signal, RNA-binding protein

The Xenopus germline is specified by inheriting a special cytoplasm, germ plasm (GP), associated with the vegetal cortex of the egg. We demonstrated that the GP was sufficient for germline specification (Tada et al., 2012). It is divided almost equally into daughter blastomeres until the 4-cell stage and is then distributed unequally to one daughter blastomere at each successive cleavage until stage 9 (Whitington and Dixon, 1975). Subsequently, it moves from the cortex to the perinuclear region and is distributed equally into two daughter primordial germ cells (PGCs). Midblastula transition (MBT), when zygotic genes begin to be expressed in most blastomeres, is observed around stage 9. However, gene expression is repressed in PGCs at MBT, so PGC specification likely occurs later (Venkatarama et al., 2010). Xpat protein, a GP component, moves from the cortex to the perinuclear region and then enters the nucleus (Machado et al., 2005). These observations suggest that some signal from GP to the nucleus is required for PGC specification.

Dead end ( $d n d$ ) encoding an RNA-binding protein with an RNA recognition motif ( $R \mathrm{RM}$ ) has been identified as a germlinespecific gene in vertebrates including zebrafish, Xenopus and mice (Weidinger et al., 2003). In Xenopus, the dnd1 transcript is a GP component and is required for PGC migration (Horvay et al., 2006). Furthermore, mouse $d n d 1$ is responsible for the Ter mutant, which causes high frequency teratoma generation in a particular genetic background (Youngren et al., 2005). Several reports have described the intracellular localization of Dead end protein; in chicks Dnd protein is present in the nuclei of PGCs and mature male germ cells (Aramaki et al., 2009). In mice, two isoforms, DND1 $\alpha$ and DND1 $\beta$, were identified and localized to the nucleus and cytoplasm when expressed in HeLa and COS-7 cells, respectively (Bhattacharya et al., 2007). In zebrafish, Dnd was localized to germ cell granules (GCGs, probably equivalent to Xenopus GP) in the perinuclear region of $\mathrm{PGCs}$ after MBT. Interestingly, this localization was dependent upon the RNA recognition motif (RRM) and nuclear export mechanism via CRM1, suggesting Dnd carried RNA from the nucleus to GCGs (Slanchev et al., 2009). Moreover, Dnd protein prevented mRNA from degradation by micro RNA targeting (Kedde et al., 2007). Thus, although Dnd might have an important role in RNA metabolism during germline development, how it functions is unclear. This study investigated the intracellular localization of Xenopus Dead end protein (Dnd1) in PGCs.

\section{Results and Conclusions}

We examined the localization of Xenopus Dnd1 in PGCs by expressing Dnd1-mCherry fusion protein in transgenic Xenopus embryos, into which a gene encoding enhanced green fluorescent protein (EGFP) tagged with a mitochondrial targeting signal had been introduced (mito-EGFP embryos) (Taguchi et al., 2012). The GP was visible in the embryos because it is enriched for mitochondria (Venkatarama et al., 2010). The mCherry signal was detected exclusively in the GP at stage 8 as previously reported (Mei et al., 2013) (Fig. 1A). In zebrafish, Dnd was localized to GCGs via the nucleus, because its expression in embryos treated

Abbreviations used in this paper: Dnd, dead end protein; GP, germ plasm; GPS, germ plasm localization signal; PGC, primordial germ cell.

\footnotetext{
*Address correspondence to: Hidefumi Orii. Department of Life Science, University of Hyogo, 3-2-1 Koto, Kamigori, Akou-gun, Hyogo 678-1297, Japan. Tel/Fax: +81-791-58-0187. E-mail: orii@sci.u-hyogo.ac.jp
}

Accepted: 20 December 2014.

ISSN: Online 1696-3547, Print 0214-6282 

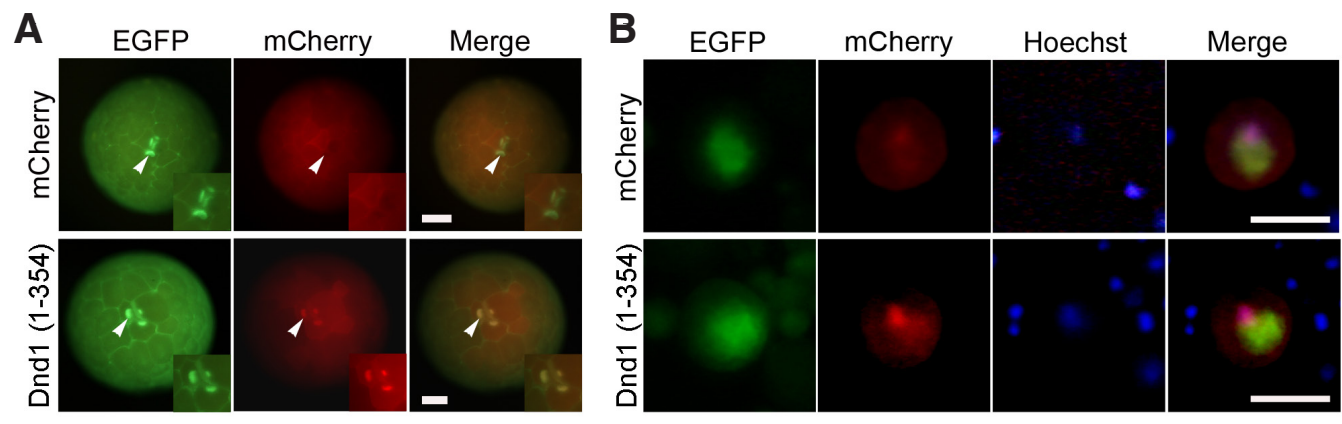

Fig. 1. Intracellular localization of Dnd1-mCherry fusion protein in primordial germ cells (PGCs). EGFP (green) and mCherry (red) signals indicate localization of GP and Dnd1, respectively. (A) Vegetal views of mito-EGFPXenopus embryo expressing mCherry or Dnd1$m$ Cherry at stage 8 . White arrowheads indicate the position of germ plasm. High magnification images of GP are shown in the insets. Scale bar $=200 \mu \mathrm{m}$. (B) Isolated PGCs expressing mCherry or Dnd1-mCherry at stage 15. The nuclei were stained with Hoechst 33242 (blue). Cells were observed under a BZ-8000 light microscope (Keyence). Scale bar, 50 um.

with leptomycin B (nuclear export inhibitor) was lost in GCGs but accumulated in the nucleus (Slanchev et al., 2009). Although we also treated all embryos with leptomycin B to exclude the possibility that Dnd1 would be localized via the nucleus, this did not appear to affect Dnd1 localization (data not shown). Because CRM1 protein involved in nuclear export is inactive until the neurula stage in Xenopus (Callanan et al., 2000), we conclude that Dnd1 does not localize to GP via the nucleus. Dnd1 appears to translocate from the cortex to the perinuclear region together with GP at the MBT.
In most embryos at stage 15, mCherry signal was detected in the nucleus or nucleus and perinuclear GP (Fig. 1B), indicating Dnd1 moved from GP to the nucleus after perinuclear translocation. Because Dnd1 is an RNA-binding protein and GP contains germline determinants (Tada et al., 2012), we speculate some RNA stored in GP before MBT might be carried to the perinuclear region and the nucleus with $D$ nd1, and trigger germline specification after MBT. Interestingly, Dnd1 and Xpat exhibit similar behavior (Machado et al., 2005).

A

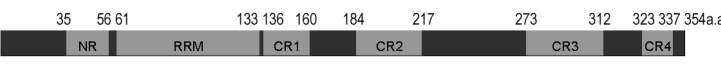

Germ Plasm $\mathrm{N} n$ localization Probability

mCherry

\section{Dnd1 (1-354) \\ Dnd1 (1-312) \\ Dnd1 (1-219)}

Dnd1 (220-312)

Dnd1 (220-285)

Dnd1 (233-312)

Dnd1 (253-312)

Dnd1 (233-252)

Dnd1 (253-285)

Dnd1 (286-312)

Dnd1 (1-232/313-354)

Dnd1 (1-60/136-354)

Dnd1 (Y72D)

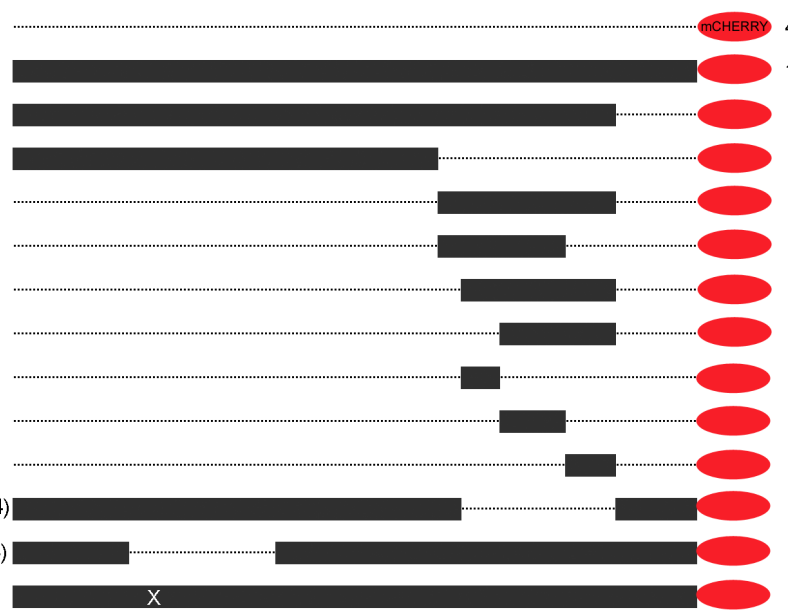

44598

257

65

101

152

45

$3 \pm 3 \%$

$91 \pm 3 \%$

$59 \pm 6 \%$

$2 \pm 2 \%$

$9 \pm 5 \%$

$44 \pm 8 \%$

$12 \pm 2 \%$

$29 \pm 2 \%$

$48 \pm 4 \%$
To identify the Dnd1 GP localization signal, we constructed deleted mutants of Dnd1 coupled with mCherry and compared their GP localization at stage 8 (Fig. 2A). Comparisons between Dnd1(1-219), Dnd1(1-312) and Dnd1(1-354) revealed Dnd1 amino acids (aa)313-354 were not involved in GP localization. The Dnd1(220-312) could localize to GP as well as Dnd1(1-354). The $\mathrm{N}$ - and $\mathrm{C}$-terminal-deleted mutants of aa220-312 were also examined. Finally, aa233-312 was identified as the region required for GP localization. Deletion of aa233-312 [Dnd1(1-232/313-354)] markedly reduced GP localization (Fig. 2B),
B

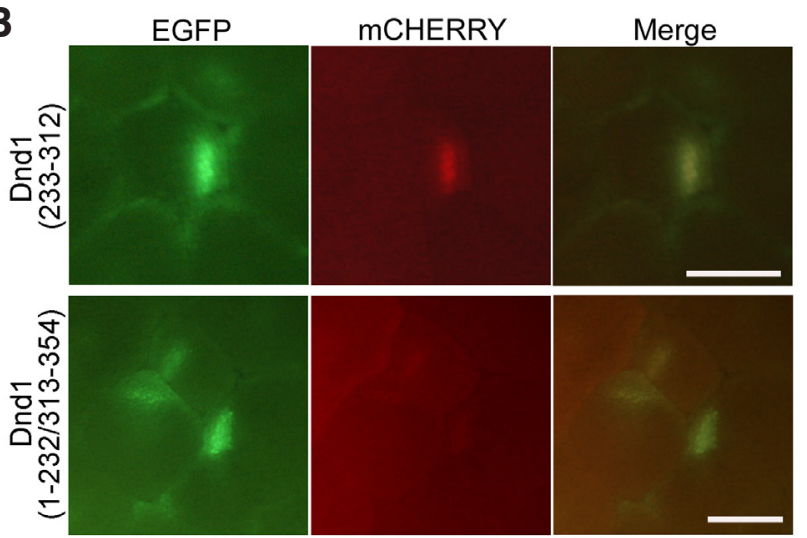

Fig. 2. Localization of the various parts of Dnd1 to the germ plasm (GP) at stage 8. (A) Schematic drawing of mCherry fused with various Dnd1 parts (left) and their localization (right). The structure of Dnd1 is based on Liu et al., (2009) (top). N-terminal region, RNA recognition motif, and C-terminal regions are indicated by NR, RRM, and CR1-4, respectively. Dnd(Y72D) shows the mutant in which Tyr at the amino acid position 72 is converted into Asp. $\mathbf{N}$ and $\mathbf{n}$ indicate the number of experiments and the total number of embryos examined, respectively. Ten to 20 embryos were examined in each experiment. Localization activity is shown as the percentage of embryos with mCherry signals in the GP among all embryos examined in each experiment. All data are presented as the mean \pm s.d. of repeated experiments. The probability value against Dnd1(1-354) (closed diamond) was calculated using two-tailed Student's t tests and a significant difference $(\mathrm{P}<0.01)$ is indicated by + . Note that aa233-312 is necessary and sufficient for GP localization and that mutants lacking RNA binding activity still localize to GP. (B) Vegetal views of mito-EGFP Xenopus embryos expressing Dnd1(233-312)-mCherry (upper) and Dnd1(1-232/313-354)-mCherry (lower) at stage 8. Scale bars $=100 \mu \mathrm{m}$. 
A

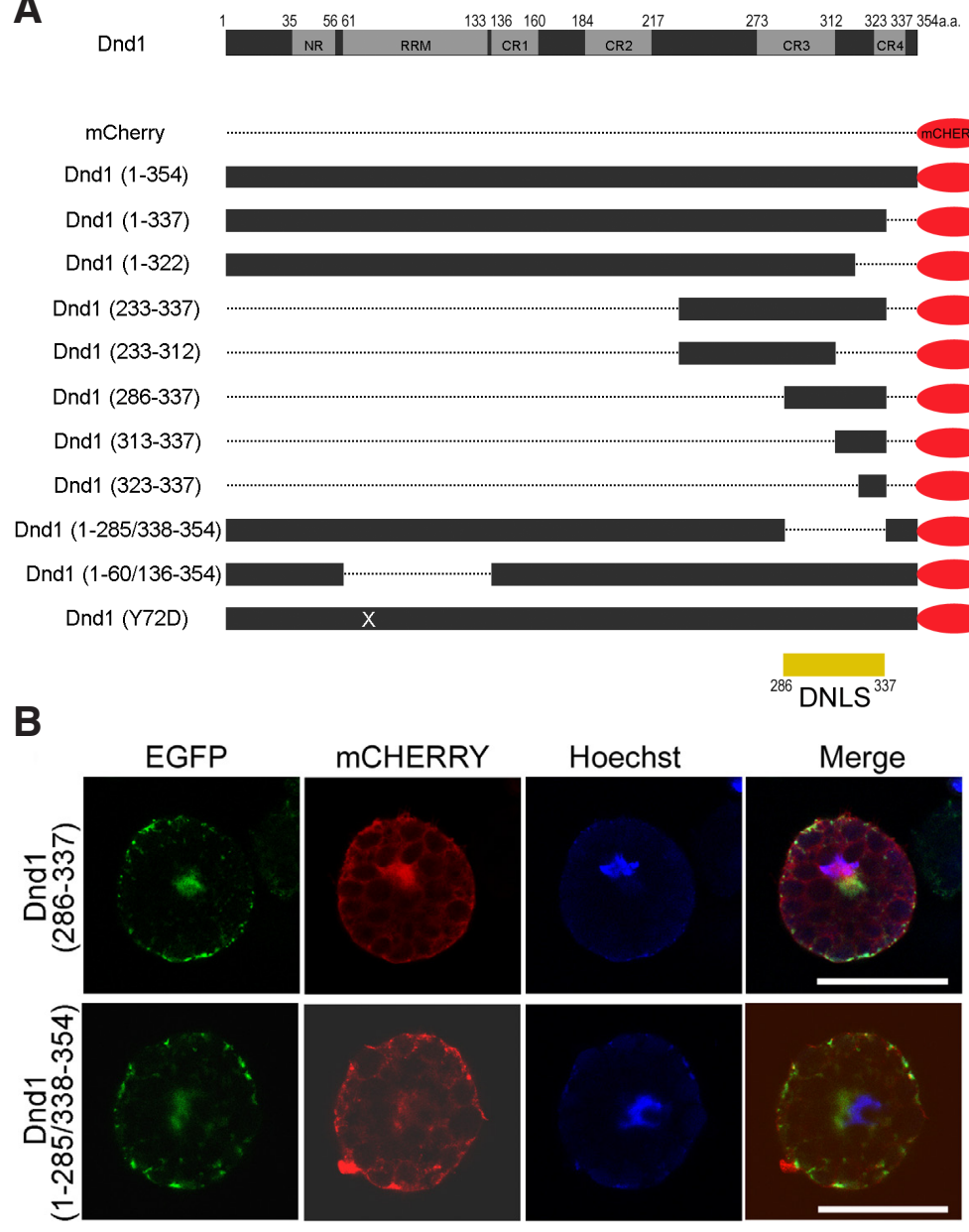

Fig. 3. Localization of the various parts of Dnd1 to the nucleus in primordial germ cells (PGCs) at stage 15. (A) Schematic drawing of mCherry fused with various Dnd1 parts (left) and their localization (right). At stage 15, PGCs were isolated from mito-EGFP embryos injected with various mRNA constructs. " $\mathrm{N}$ " and " $\mathrm{n}$ " indicate the total numbers of experiments labout 10 embryos per experiment) and PGCs examined, respectively. Nuclear localization activity is shown as the percentage of PGCs with a nuclear mCherry signal among all PGCs examined. The probability value against Dnd1 (1-354) (closed diamond) was calculated using Fisher's test and a statistically significant difference $(P<0.01)$ is indicated by + . Note that aa286-337 is necessary and sufficient for nuclear localization and that mutants lacking RNA binding activity still localize to nucleus. (B) PGCs isolated from mito-EGFP embryos expressing Dnd1(286-337)-m Cherry (upper) and Dnd1(1-285/338-354)-mCherry (lower) at stage 15. The nuclei were stained with Hoechst 33242 (blue). Cells were abserved under a TCS SP8 confocal microscope (Leica). Scale bars, 50 um.

Interestingly, Dnd1(1-285/338-337) showed perinuclear GP localization, but not nuclear localization (Fig. 3B).

GPS (aa233-312) and DNLS (aa286-337) are overlapping but distinct regions (Fig. 4). Although GPS/DNLS-mCherry [Dnd1(233-337)] localized to GP at stage 8 and the nucleus at stage 15, DNLS-mCherry-GPS (DNLS and GPS are located separately) localized to GP at stages $8 / 15$, but not the nucleus at stage 15 . Unfortunately we do not know the localization of GPS-mCherry-DNLS, because we failed to construct it. In addition, we compared DNLS with a nuclear localization signal (NLS) of simian virus 40 (SV40) (Lanford et al., 1986), in relation to GPS. mCherry-NLS did not localize to the nucleus or GP at stage 8 , but localized to the nucleus at stage 15. However, GPS-mCherryNLS and GPS/DNLS-mCherry localized to GP at stage 8 and the nucleus at stage 15 . Although both DNLS and NLS function as a nuclear localization signal after MBT, DNLS does not seem to function alone if it is present in GP at MBT. Together with the finding that DNLS overlaps with GPS, interaction between GPS and DNLS might be important for transition of Dnd1 localization from GP to the nucleus at MBT. Activated GPS (probably bound to some factors) might be a prerequisite for nuclear localization via DNLS afterward. Further experiments are needed to know how they interact. Thus, DNLS as well as NLS does not seem to function before MBT (Saka et al., 2007).

Finally, to examine whether DNLS was active in a PGC-specific manner, we injected Dnd1(1-354) or Dnd1(286-337) into the equatorial region of a fertilized egg (Fig. 5). mCherry signal was observed throughout embryonic somatic cells at stage 15. In contrast, NLS-mCherry localized to the nucleus in somatic cells as previously reported (Saka et al., 2007), indicating that DNLS functions in a PGC-specific manner.

In zebrafish, Dnd localizes to GCGs via the nucleus after MBT, but the behavior of Dnd before this stage is unknown (Slanchev et al., 2009). Here, we demonstrated that Dnd1 initially localized to and Dnd 1 (Y72D) showed high activity ( $68 \%$ and $81 \%$, respectively). DNLS has no similarity with other nuclear localization signals. 
A

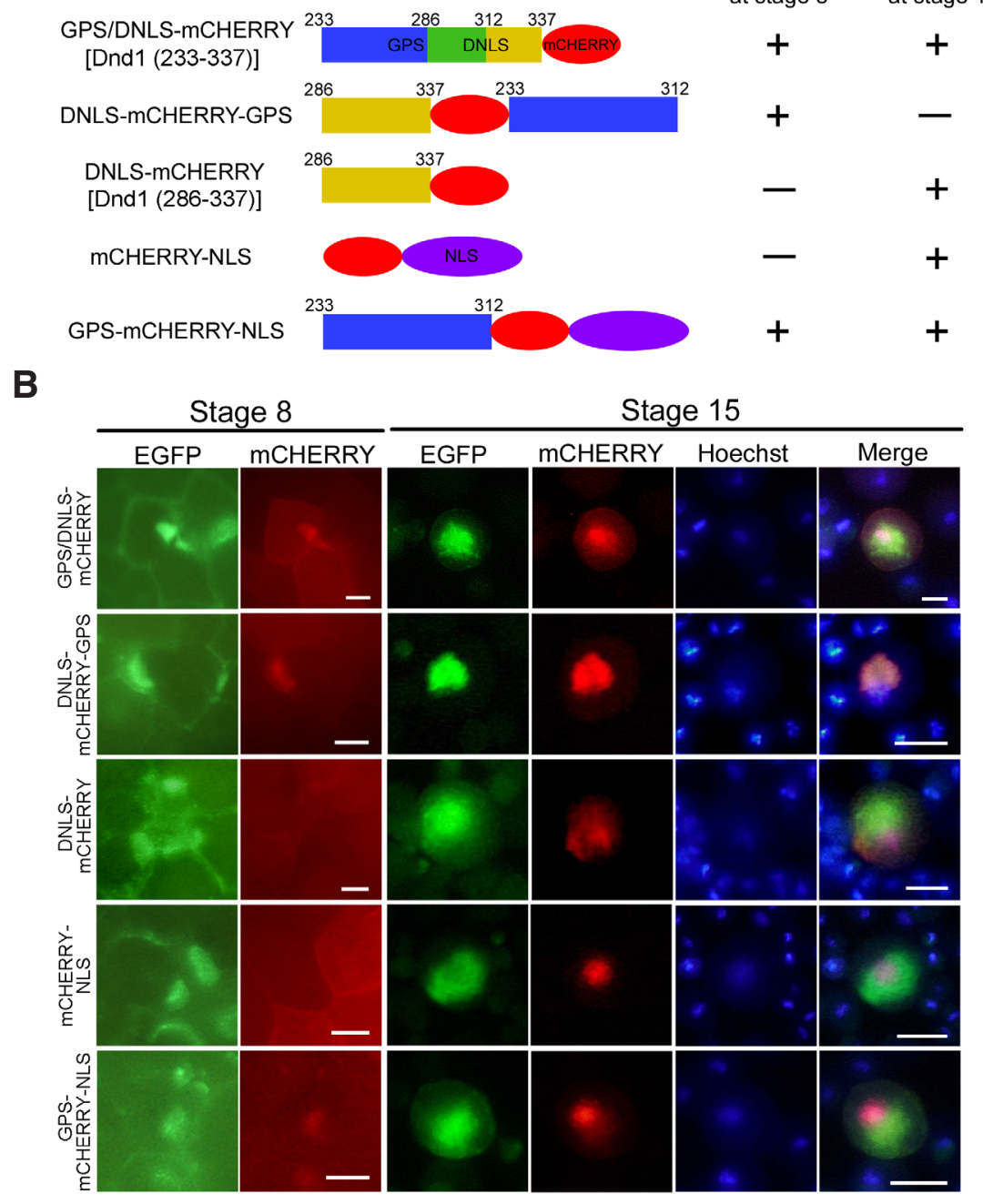

Fig. 4. Relationship of the germ plasm localization signal (GPS) and nuclear localization signals. (A) Schematic drawing of mCherry fused with GPS and SV4ONLS or DNLS and summary of the results. Germ plasm and nuclear localization activities are shown by the same way as Fig. 2 and Fig. 3, respectively. (B) Intracellular localization of various fusion proteins. Vegetal views of the injected embryos at stage 8 (left). PGCs isolated at stage 15 were stained with Hoechst 33242 (blue) and observed under a BZ-8000 light microscope (Keyence) (right). Scale bars, 100 um (stage 8) and 50 um (stage 15). Nuclei were stained with Hoechst 33242 (blue).
GP, moved to the perinuclear region and then entered the nucleus after MBT. Dnd1 might also move outside the nucleus after that event. Therefore, the Dead end protein might function as a shuttle protein similar to other RNA-binding proteins with RRMs including $\mathrm{Hu}$ proteins (Hinman and Lou, 2008). Of note, mouse Dnd1 was localized to GCGs when expressed in zebrafish PGCs (Slanchev et al., 2009). Surprisingly, zebrafish Dnd aa322-411 corresponding to around Xenopus Dnd1 GPS/DNLS localized to GP at stage 8 and the nucleus at stage 15 when expressed in Xenopus PGCs (Fig. 6). There is a little similarity in amino acid sequence between them (Fig. 6A). These suggest that translocation mechanisms of Dnd are conserved among vertebrates. The ATPase activity detected in zebrafish Dnd aa322-411 (Liu and Collodi, 2010) may be involved in the translocation.

Xenopus GP contains germline determinants (Tada et al., 2012). Xenopus Dnd1 moves from the cortex to the perinuclear region together with GP and then enters the nucleus. Then, germline specification occurs (Lai et al., 2012). Together with the finding that Dnd1 is an RNA-binding protein, we speculate that Dnd1 carries RNAs into the nucleus to trigger germline specification. It might also have nuclear functions including RNA processing. Although the mRNA targets of DND1 have been identified in human embryonic stem cells (Zhu et al., 2011), it remains to know the localization and function of the homologues in Xenopus germline development. To understand germline specification, it is critical to clarify the functions of Dnd1, including identification of its target RNA and interacting proteins.

\section{Materials and Methods}

\section{Xenopus embryos}

Adult wild-type Xenopus laevis toads were purchased commercially and maintained at $22{ }^{\circ} \mathrm{C}$ in circulatory water tanks. A transgenic mito-EGFP Xenopus line was generated recently in our laboratory (Taguchi et al., 2012). Eggs from mito-EGFP female Xenopus were fertilized in vitro with wildtype spermatozoa, injected with mRNA and then allowed to develop at $16^{\circ} \mathrm{C}$ as described (Kataoka etal., 2006). Embryos were staged according to Nieuwkoop and Faber (1994). For observation at stage 8 , embryos were treated with $200 \mathrm{ng} /$ $\mathrm{mL}$ of leptomycin $\mathrm{B}$ (Enzo Life Sciences, Farmingdale, $\mathrm{NH}$,

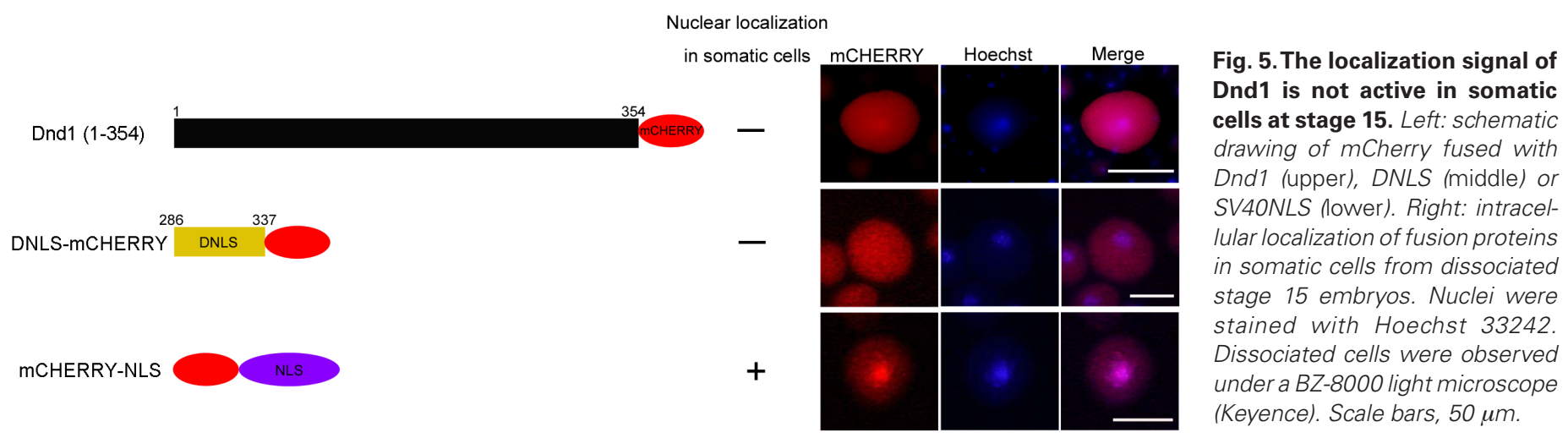




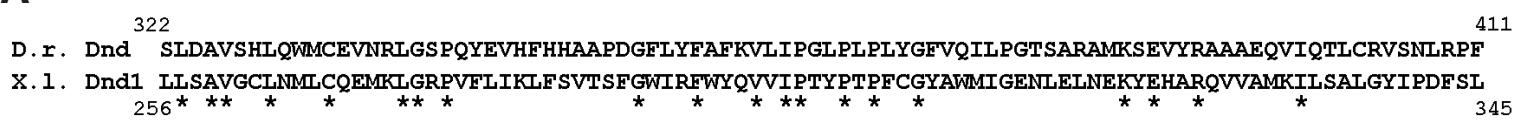

B

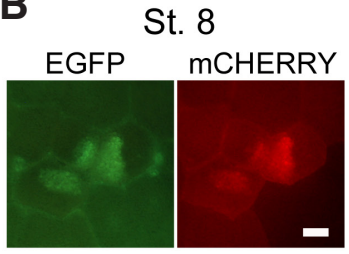

St. 15

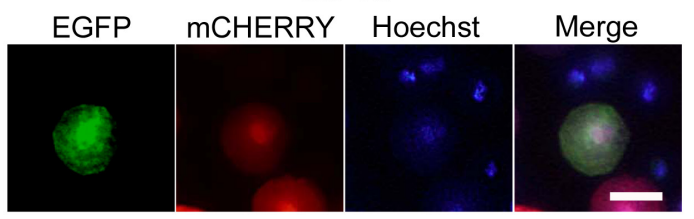

Fig. 6. Intracellular localization of zebrafish Dnd fragment in Xenopus primordial germ cells (PGCs). (A) Zebrafish (D.r.) Dnd aa322-411 used in this experiment is compared with Xenopus (X.I.) Dnd1 aa256-345. Asterisks indicate identical amino acids. (B) Intracellular localization of zebrafish Dnd aa322-411-mCherry [Dnd(322-411)] in Xenopus PGCs at stages 8 and 15. Images at stage 15 were purchased using a BZ-8000 light microscope (Keyence). Scale bars, $100 \mu \mathrm{m}$ at stages 8 and $50 \mu \mathrm{m}$ at stage 15.

USA) continuously. For observation of PGCs at stage 15, embryos were treated with $200 \mathrm{ng} / \mathrm{mL}$ of leptomycin B from stage 10 .

\section{Preparation of constructs}

An open reading frame of $d n d 1 \mathrm{cDNA}$ was amplified by polymerase chain reaction (PCR) with the following set of primers: 5'-CTTTTTGCAGGATCCATGGAGCTGTCAGAC-3' (the sequence corresponding to the pCS2 vector is underlined) and 5'-CTTGATGATGGCCATCAGGGCATTCCTTGC-3' (the sequence corresponding to the $\mathrm{N}$-terminal of the $\mathrm{mCherry} \mathrm{protein} \mathrm{is} \mathrm{underlined)}$ using Xenopus laevis ovarian cDNA as a template, cloned finally into the pCS2-mCherry-DEADSouth 3' untranslated region (UTR) vector (Kataoka et al., 2006) using In-Fusion technology (Clontech, Palo Alto, CA, USA). This construct pCS2-xDND-mCherry-DS 3' UTR (xDND1-354) encodes a fusion protein of Xenopus Dnd 1 followed by mCherry. In our cloned Xenopus dnd 1 cDNA, the Asn and Met at aa 150 and 155 were changed to Thr and Leu, respectively, compared with the Dnd1 sequence registered in DDBJ/ GenBank/EMBL (accession no. AY971581). The differences probably arose from genetic polymorphism, because the same substitutions were found in several clones from independent PCR runs. Various mutant and deletion constructs were made from pCS2-xDND-mCHRY-DS 3' UTR by PCR and In-Fusion technology (Clontech). A partial fragment of $d n d$ was amplified by PCR with total DNA extracted from zebrafish. A conventional NLS (from SV40) was also amplified by PCR with phGFP105Sse-NLS (Yagisawa et al., 2002). All constructs were confirmed by sequencing.

\section{Preparation and microinjection of $m R N A$}

The constructs were linearized by digestion with Notl and used as templates for mRNA synthesis with an mMESSAGE mMACHINE SP6 kit (Ambion, Austin, TX, USA). The mRNAs were dissolved in water $(0.1 \mu \mathrm{g} / \mu \mathrm{L})$ and $9.2 \mathrm{~nL}$ aliquots were microinjected into the cortical region at the vegetal pole of fertilized eggs using a Nanoject II microinjector (Drummond Scientific, Broomall, PA, USA). For expression in other than PGCs, constructs were injected into the equatorial region of fertilized eggs.

\section{PGC isolation and observation}

Embryos at stage 8 were fixed in $2 \%$ paraformaldehyde/0.5 M NaCl/0.1 M MOPS buffer ( $\mathrm{pH} 7.5$ ) at $4{ }^{\circ} \mathrm{C}$ overnight, settled in Dulbecco's phosphatebuffered saline without $\mathrm{Ca}^{2+}$ and $\mathrm{Mg}^{2+}$ (PBS-) at $4{ }^{\circ} \mathrm{C}$ for 2 days and then observed using an MZ16F fluorescence stereomicroscope (Leica Microsystems, Wetzlar, Germany). Embryos at stage 15 were transferred to $70 \%$ PBS- for dissociation. PGCs were collected manually using a micropipette, fixed and treated in the same way. PGCs were stained for nuclei with $0.3 \mu \mathrm{g} /$ mL Hoechst 33242 (Sigma-Aldrich, St Louis, MO, USA), rinsed in PBS-and mounted in 50\% glycerol/PBS-. Cells were observed under an LSM510 confocal laser scanning microscope (Carl Zeiss, Oberkochen, Germany) or a TCS SP8 (Leica Microsystems), or a BZ-8000 light microscope equipped with BZ-II software (Keyence, Osaka, Japan). We judged the GP and nuclear localization of Dnd1 constructs only by visually comparing with EGFP and Hoechst signals, respectively.

\section{Statistical analyses}

For localization of Dnd1-derived proteins to the GP in stage 8 embryos, we calculated the ratio of embryos in which the mCherry signal was localized to the GP to the total number embryos in each experiment using the same cohort of embryos. The mean \pm standard deviation (s.d.) of ratios from several repeated experiments were subjected to statistical analyses using Student's $t$ test. For localization of Dnd1 derivative proteins in PGCs at stage 15, the ratios of indicated PGCs to total PGCs in several experiments were calculated and were subjected to statistical analyses using Fisher's test.

\section{Acknowledgements}

We are grateful to Drs. Takanori Ikenaga and Hitoshi Yagisawa for the zebrafish and phGFP105Sse-NLS, respectively. We also thank members of our laboratory, especially Dr. Makoto Mochii, for their support and fruitful discussions.

\section{References}

ARAMAKI, S., KUBOTA, K., SOH, T., YAMAUCHI, N. and HATTORI, M. (2009) Chicken dead end homologue protein is a nucleoprotein of germ cells including primordial germ cells. J. Reprod. Dev. 55: 214-218.

BHATTACHARYA, C., AGGARWAL, S., ZHU, R., KUMAR, M., ZHAO, M., MEISTRICH, M.L. and MATIN, A. (2007). The mouse dead-end gene isoform $\alpha$ is necessary for germ cell and embryonic viability. Biochem. Biophys. Res. Commun. 355: 194-199.

CALLANAN, M., KUDO, N., GOUT, S., BROCARD, M., YOSHIDA, M., DIMITROV, S and KHOCHBIN, S. (2000). Developmentally regulated activity of CRM1/XPO1 during early Xenopus embryogenesis. J. Cell Sci. 113: 451-459.

HINMAN, M.N. and LOU, H. (2008). Diverse molecular functions of Hu proteins. Cell Mol. Life Sci. 65: 3168-3181.

HORVAY, K., CLAUSSEN, M., KATZER, M., LANDGREBE, J. and PIELER, T. (2006). Xenopus Dead end mRNA is a localized maternal determinant that serves a conserved function in germ cell development. Dev. Biol. 291: 1-11.

KATAOKA, K., YAMAGUCHI, T., ORII, H., TAZAKI, A., WATANABE, K. and MOCHII, M. (2006). Visualization of the Xenopus primordial germ cells using a green fluorescent protein controlled by cis elements of the 3' untranslated region of DEADSouth gene. Mech. Dev. 123: 746-760.

KEDDE, M., STRASSER, M.J., BOLDAJIPOUR, B., OUDE VRIELINK, J.A.F., SLANCHEV, K., LE SAGE, C., NAGEL, R., VOORHOEVE, P.M., VAN DUIJSE, J., ØROM, U.A., LUND, A.H., PERRAKIS, A., RAZ, E. and AGAMI, R. (2007). RNA-binding protein Dnd1 inhibits microRNA access to target mRNA. Cell 131: 1273-1286.

LAI, F., SINGH, A. and KING, M.L. (2012). Xenopus Nanos1 is required to prevent endoderm gene expression and apoptosis in primordial germ cells. Development 139: $1476-1486$.

LANFORD, R.E., KANDA, P. and KENNEDY, R.C. (1986). Induction of nuclear transport with a synthetic peptide homologous to the SV40 antigen transport signal. Cell 46: 575-582

LIU, L., HONG, N., XU, H., LI, M., YAN, Y., PURWANTI, Y., YI, M., LI, Z., WANG, L. and HONG, Y. (2009). Medaka dead end encodes a cytoplasmic protein and 
identifies embryonic and adult germ cells. Gene Expression Patt. 9: 541-548.

LIU, W. and COLLODI, P. (2010). Zebrafish dead end possesses ATPase activity that is required for primordial germ cell development. FASEB J. 24: 2641-2650.

MACHADO, R.J., MOORE, W., HAMES, R., HOULISTON, E., CHANG, P., KING, M.L. and WOODLAND, H.R. (2005). Xenopus Xpat protein is a major component of germ plasm and may function in its organisation and positioning. Dev. Biol. 287: 289-300.

MEI, W., JIN, Z., LAI, F., SCHWEND, T., HOUSTON, D.W., KING, M.L. and YANG, J. (2013). Maternal Dead-End1 is required for vegetal cortical microtubule assembly during Xenopus axis specification. Development 140: 2334-2344.

NIEUWKOOP, P.D. and FABER, J. (1994). Normal table of Xenopus laevis (Daudin): A systematical and chronological survey of the development from the fertilized egg till the end of metamorphosis. New York and London: Garland Pub.

SAKA, Y., HAGEMANN, A.I., PIEPENBURG, O. and SMITH, J.C. (2007). Nuclear accumulation of Smad complexes occurs only after the midblastula transition in Xenopus. Development 134: 4209-4218.

SLANCHEV, K., STEBLER, J., GOUDARZI, M., COJOCARU, V., WEIDINGER, G. and RAZ, E. (2009). Control of Dead end localization and activity-implications for the function of the protein in antagonizing miRNA function. Mech. Dev. 126: 270-277.

TADA, H., MOCHII, M., ORII, H. and WATANABE, K. (2012). Ectopic formation of primordial germ cells by transplantation of the germ plasm: Direct evidence for germ cell determinant in Xenopus. Dev. Biol. 371: 86-93.

TAGUCHI, A., TAKII, M., MOTOISHI, M., ORII, H., MOCHII, M. and WATANABE, K. (2012). Analysis of localization and reorganization of germ plasm in Xenopus transgenic line with fluorescence-labeled mitochondria. Dev. Growth Differ. 54:
767-776.

VENKATARAMA, T., LAI, F., LUO, X., ZHOU, Y., NEWMAN, K. and KING, M.L. (2010) Repression of zygotic gene expression in the Xenopus germline. Development 137: 651-660.

WEIDINGER, G., STEBLER, J., SLANCHEV, K., DUMSTREI, K., WISE, C., LOVELLBADGE, R., THISSE, C., THISSE, B. and RAZ, E. (2003). dead end, a nove vertebrate germ plasm component, is required for zebrafish primordial germ cel migration and survival. Curr. Biol. 13: 1429-1434.

WHITINGTON, P.M. and DIXON, K.E. (1975). Quantitative studies of germ plasm and germ cells during early embryogenesis of Xenopus laevis. J. Embyol. Exp. Morph. 33: 57-74.

YAGISAWA, H., YAMAGA, M., OKADA, M., SASAKI, K. and FUJII, M. (2002). Regulation of the intracellular localization of phosphoinositide-specific phospholipase C81. Advan. Enzyme Regul. 42: 261-284.

YAMAGUCHI, T., TAGUCHI, A., WATANABE, K. and ORII, H. (2013). DEADSouth protein localizes to germ plasm and is required for the development of primordial germ cells in Xenopus laevis. Biol. Open 2: 191-199.

YOUNGREN, K.K., COVENEY, D., PENG, X., BHATTACHARYA, C., SCHMIDT, L.S., NICKERSON, M.L., LAMB, B.T., DENG, J.M., BEHRINGER, R.R., CAPEL, B., RUBIN, E.M., NADEAU, J.H. and MATIN, A. (2005). The Ter mutation in the dead end gene causes germ cell loss and testicular germ cell tumours. Nature 435: 360-364

ZHU, R., IACOVINO, M., MAHEN, E., KYBA, M. and MATIN, A. (2011). Transcripts that associate with the RNA binding protein, DEAD-END (DND1), in embryonic stem (ES) cells. BMC Mol. Biol. 12: 37. 


\section{Further Related Reading, published previously in the Int. J. Dev. Biol.}

Identification of promoter elements responsible for gonad-specific expression of zebrafish Deadend and its application to ovarian germ cell derivation Ten-Tsao Wong, Abraham Tesfamichael and Paul Collodi Int. J. Dev. Biol. (2013) 57: 767-772

Germes is involved in translocation of germ plasm during development of Xenopus primordial germ cells

Takeshi Yamaguchi, Ayaka Taguchi, Kenji Watanabe and Hidefumi Orii Int. J. Dev. Biol. (2013) 57: 439-443

Visualization of primordial germ cells in vivo using GFP-nos1 3'UTR mRNA

Taiju Saito, Takafumi Fujimoto, Shingo Maegawa, Kunio Inoue, Minoru Tanaka, Katsutoshi Arai and Etsuro Yamaha

Int. J. Dev. Biol. (2006) 50: 691-700

\section{Primordial germ cell migration}

Kathleen Molyneaux and Christopher Wylie

Int. J. Dev. Biol. (2004) 48: 537-543

Twenty years of research on primordial germ cells.

M De Felici

Int. J. Dev. Biol. (2001) 45: 519-522

5 yr ISI Impact Factor $(2013)=2.879$
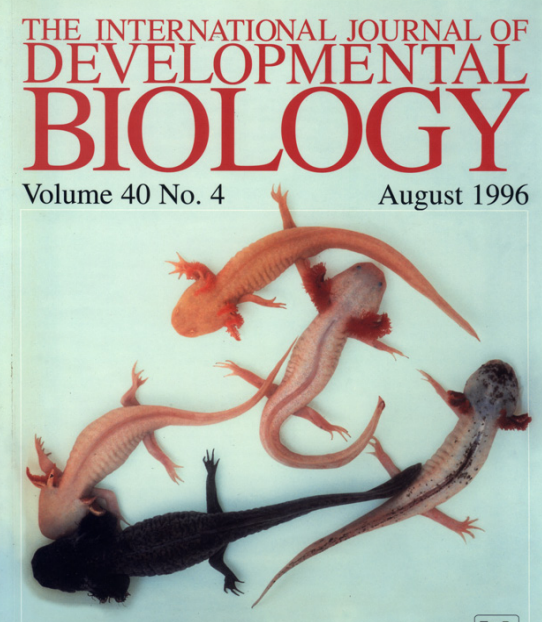

Developmental Biology of Urodeles
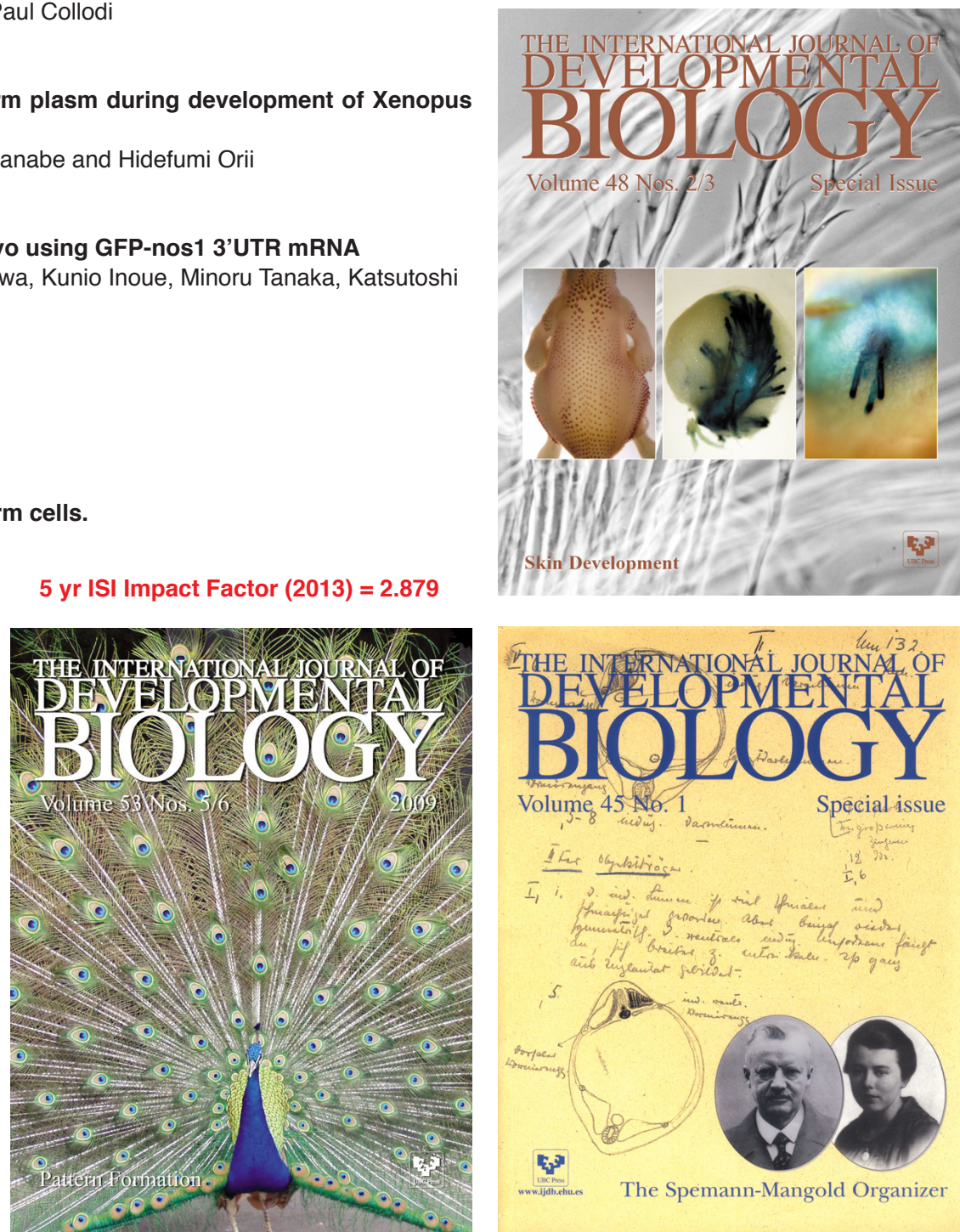

Volume 45 No. 1

Special issue
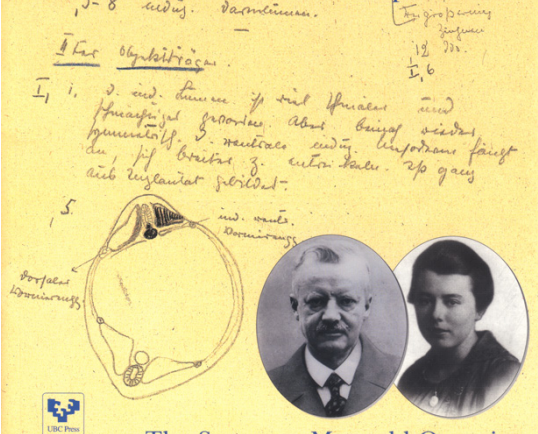

5

The Spemann-Mangold Organizer 